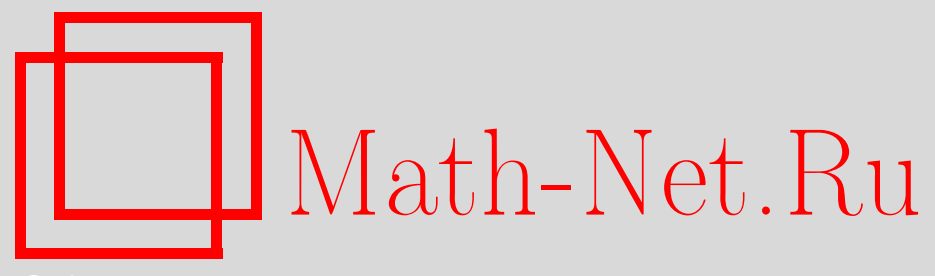

Е. Бакаев, Обход многоугольника, Квант, 2020, номер 8, $27-$ 31

DOI: https://doi.org/10.4213/kvant20200802

Использование Общероссийского математического портала Math-Net.Ru подразумевает, что вы прочитали и согласны с пользовательским соглашением http://www . mathnet.ru/rus/agreement

Параметры загрузки:

IP : 34.227 .88 .159

26 апреля 2023 г., 07:50:16

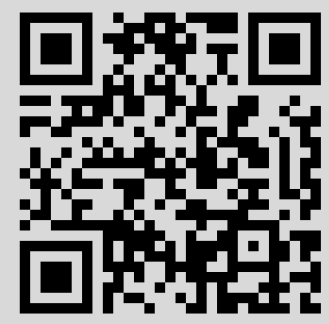




\section{Обход многоугольника}

E.

КОНКУРСЕ ИМЕНИ А.П.САВИНА

в «Кванте» №12 за 2019 год предлагалось решить следующую задачу для некоторых конкретных значений $n$ (автор задачи - А.Перепечко):

Барон Мюнхгаузен огородил свои владения забором в форме п-угольника. Он утверждает, что каждый внутренний угол этого п-угольника либо меньше $10^{\circ}$, либо больше $350^{\circ}$. Может ли барон быть прав? Решите задачу для а) $n=10$; б) $n=11$; в) $n=101$.

Решим эту задачу для всех натуральных $n$. Будем называть углы многоугольника, меньшие $10^{\circ}$, маленькими, а бо́льшие $350^{\circ}-$ большими. Итак, нас интересуют многоугольники, в которых $a$ маленьких углов и $b$ больших, $a+b=n$.

\section{Четные $n$}

Начнем с наименьших $n$. Понятно, что для $n=3$ такого многоугольника не существует. Для $n=4$ пример есть (рис.1, $a$ ). Еще порисовав многоугольники, можно додуматься, как устроена серия примеров для всех четных $n$ (рис.1, 6, в). Здесь и далее будем отмечать маленькие углы фиолетовым цветом, а углы, дополняющие большие углы до полного угла, - зеленым.

Упражнение 1. Докажите, что в шестиугольнике на рисунке 1,6 выполняется равенство $\gamma=\left(\beta_{1}+\beta_{2}\right)-\left(\alpha_{1}+\alpha_{2}+\alpha_{3}\right)$.
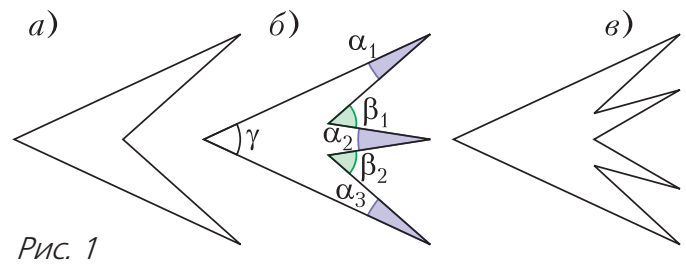

DOI: https://doi.org/10.4213/kvant20200802
Аналогично тому, как это получается в упражнении 1 , в случае $(2 k+2)$-угольника такой угол будет равен

$\gamma=\left(\beta_{1}+\beta_{2}+\ldots+\beta_{k}\right)-\left(\alpha_{1}+\alpha_{2}+\ldots+\alpha_{k+1}\right)$.

Это легко доказать с помощью теоремы о сумме углов $n$-угольника (которая гласит, что сумма углов любого $n$-угольника равна $\left.180^{\circ} \cdot(n-2)\right)$. Либо же можно представить, что мы поворачиваем верхнюю сторону (точнее, луч - идущее вдоль стороны направление) на угол $\alpha_{1}$ против часовой стрелки, потом на $\beta_{1}$ по часовой, потом на $\alpha_{2}$ против часовой стрелки и т.д. (рис.2).

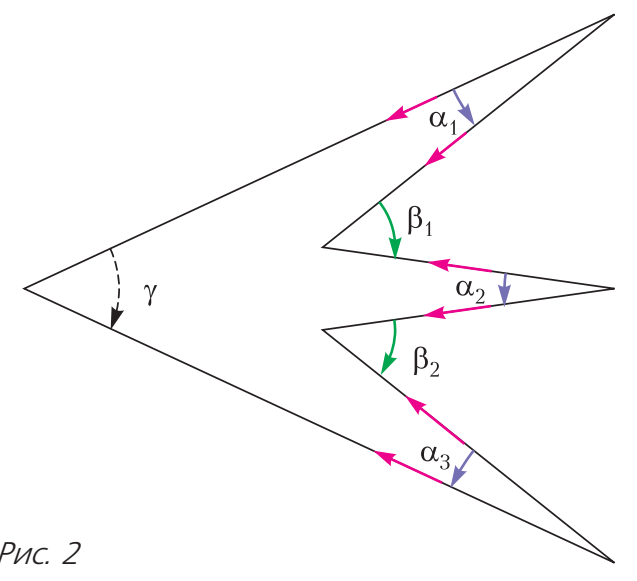

В итоге верхняя сторона повернется на угол $\gamma$ по часовой стрелке, отсюда и получаем равенство (1).

Упражнение 2. Придумайте примеры для $n=6$ и $n=8$ вида, отличного от показанного на рисунке 1.

\section{Перестройка}

Можно доказать существование примеpa, не предъявляя его явно: надо показать, как добавить к подходящему примеру две вершины так, чтобы снова получился подходящий. Тогда из подходящего примера 
для $n=4$ получится бесконечная цепочка примеров для всех четных $n$.

Возможна такая «перестройка» многоугольника: возьмем один из его маленьких углов и вырежем из него параллелограмм, как изображено на рисунке 3. Один ма-

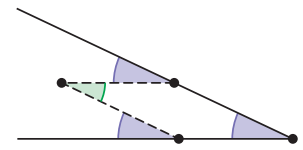

Pис. 3

ленький угол превратится в два равных ему маленьких и один большой.

Упражнение 3. Придумайте другую «перестройку», добавляющую к многоугольнику две вершины.

Итак, для многоугольников с четным количеством сторон есть серия примеров. Осталось понять, при каких нечетных $n$ существуют подходящие $n$-угольники. Рассмотрим среди всех таких $n$ наименьшее, обозначим его $M$. Тогда для всех нечетных $n$, бо́льших $M$, подходящий пример можно будет получить, нужное число раз повторив «перестройку» (также надо будет убедиться в наличии хотя бы одного маленького угла в исходном примере, чтобы «перестройку» можно было осуществить). Осталось найти $M$.

В приведенном выше рассуждении скрылась неточность: такого наименьшего $n$ может и не существовать - если подходящих примеров для нечетных $n$ нет вовсе. Но сейчас мы такой пример приведем.

\section{Первый пример}

Вернемся к конструкции на рисунке 2. При $\gamma=180^{\circ}$ такой «четноугольник» превратится в «нечетноугольник» (рис.4). Для

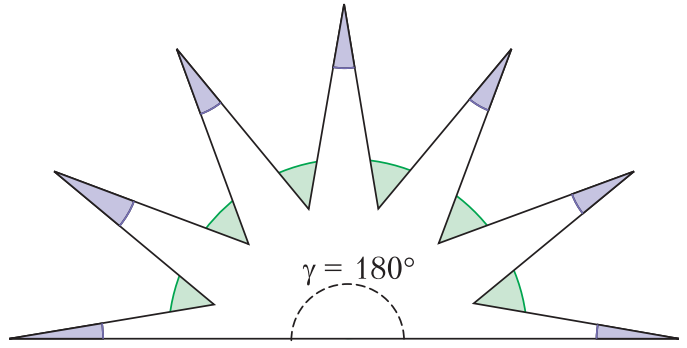

Pnc. 4 какого наименьшего $n$ устроенный таким образом пример существует? Сумма углов $\beta_{1}+\beta_{2}+\ldots+\beta_{k}$ должна быть больше $180^{\circ}$, но все слагаемые в сумме меньше $10^{\circ}$, значит, их хотя бы 19. Тогда всего вершин не менее $19+20=39$.

Нетрудно понять, что нужные значения углов подобрать возможно. Итак, пример на $n=39$ получен, а значит, $M \leq 39$.

\section{Первая оценка}

Некоторый пример получен, теперь давайте получим какую-нибудь оценку на количество сторон подходящего многоугольника. Снова будем рассматривать направления, но иначе. Начнем обход границы многоугольника против часовой стрелки и будем следить за тем, как меняется направление, в котором мы движемся.

Так как в подходящем многоугольнике каждый угол либо маленький, либо большой, то при переходе на очередную сторону направление, в котором мы движемся, меняется на почти противоположное. Затруднений с примером для четного $n$ как раз и не возникает - при обходе направление меняется на почти противоположное четное количество раз и становится исходным. В случае же нечетного $n$ нам потребуется оценить возможный вклад этих «Почти».

Можно считать, что направлениям соответствуют лучи, выходящие из одной точки. На рисунках $5, a$ и $6, a$ показаны обходы многоугольников, а на рисунках 5,б и 6,б начала тех же лучей-направлений обхода перенесены в одну точку.

Будем следить за тем, на какой угол изменяется направление. Некоторые повороты происходят по часовой стрелке, некоторые - против часовой. Направление
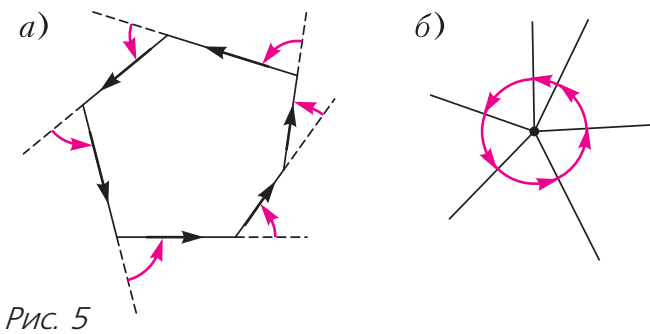

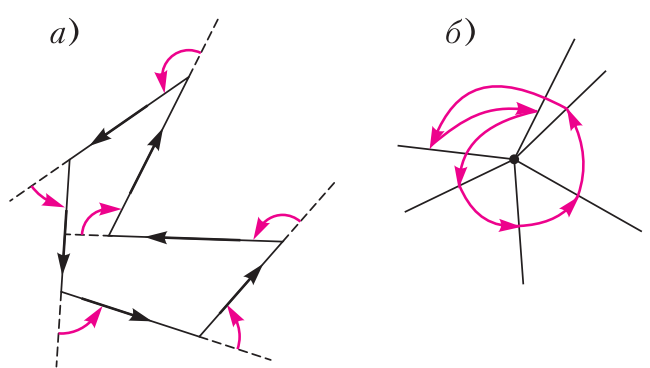

Pис. 6

против часовой стрелки будем считать положительным. Какое-то из направлений выбрано «нулевым», т.е. началом отсчета. Таким образом, направлению соответствует число (величина отклонения от начала отсчета) и при изменении направления число меняется.

При проходе через маленький угол $\alpha_{i}$ направление поворачивается на $\left(180^{\circ}-\alpha_{i}\right)$ против часовой стрелки (рис.7, $a$ ), т.е. увеличивается на $\left(180^{\circ}-\alpha_{i}\right)$. А при проходе

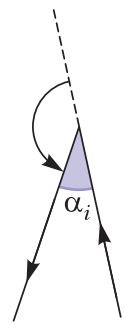

a)

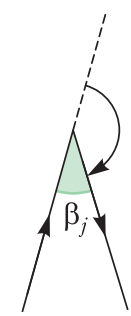

б)
Pис. 7

через большой угол $\left(360^{\circ}-\beta_{j}\right)$ оно поворачивается на $\left(180^{\circ}-\beta_{j}\right)$ по часовой стрелке (рис.7,б), т.е. уменьшается на $\left(180^{\circ}-\beta_{j}\right)$. Тогда суммарное изменение направлений будет равно

$$
\begin{gathered}
S=\left(180^{\circ}-\alpha_{1}\right)+\left(180^{\circ}-\alpha_{2}\right)+\ldots+\left(180^{\circ}-\alpha_{a}\right)- \\
-\left(180^{\circ}-\beta_{1}\right)-\left(180^{\circ}-\beta_{2}\right)-\ldots-\left(180^{\circ}-\beta_{b}\right)= \\
=180^{\circ} \cdot(a-b)-\left(\alpha_{1}+\alpha_{2}+\ldots+\alpha_{a}\right)+ \\
+\left(\beta_{1}+\beta_{2}+\ldots+\beta_{b}\right)=180^{\circ} \cdot(a-b)-A+B,(2)
\end{gathered}
$$

где через $A$ обозначена сумма $\left(\alpha_{1}+\alpha_{2}+\ldots\right.$ $\left.\ldots+\alpha_{a}\right)$, через $B-$ сумма $\left(\beta_{1}+\beta_{2}+\ldots+\beta_{b}\right)$.

Так как путь замыкается, то после $n$ поворотов направление станет таким же, как было сначала, а значит, суммарное изменение направлений $S$ должно быть кратно $360^{\circ}$. Кроме того, $a-b \neq 0$, поскольку $a+b$ нечетно. Тогда из равенства (2) получаем $|A-B| \geq 180^{\circ}$, значит, в сумме $A$ или в сумме $B$ хотя бы 19 слагаемых и $n \geq 19$. Соответственно, и $M \geq 19$.

\section{Существует ли пример на 19?}

Пока что между оценкой и примером имеется «зазор» от 19 до 39.

Существует ли пример с 19 вершинами? Если существует, то что про него можно понять из полученной нами оценки? В одной из сумм $A$ и $B$ должно быть 19 слагаемых, в другой ни одного. Значит, все повороты должны быть в одну сторону (пусть против часовой стрелки) и углы между соседними сторонами должны быть в среднем по $\frac{180^{\circ}}{19}$. Начнем рисовать такой многоугольник (рис.8).

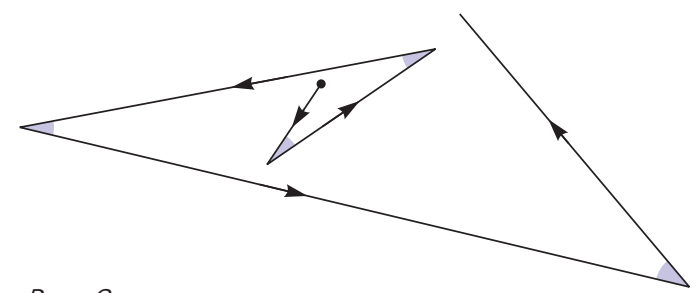

Pис. 8

Кажется, что мы движемся «по спирали» и вряд ли получится такой многоугольник замкнуть...

В оценке мы пока что не использовали то, что это многоугольник; мы опирались только на то, что это замкнутая ломаная. Напомним разницу: граница многоугольника - это, по определению, замкнутая ломаная без самопересечений. Разрешив самопересечения, можно ли нарисовать подходящую замкнутую 19-звенную ломаную? Да, пример такой замкнутой ломаной привести нетрудно. Рассмотрим правильный 19-угольник. Между двумя его самыми длинными диагоналями, выходящими из одной вершины, угол $\frac{180^{\circ}}{19}$. Так 


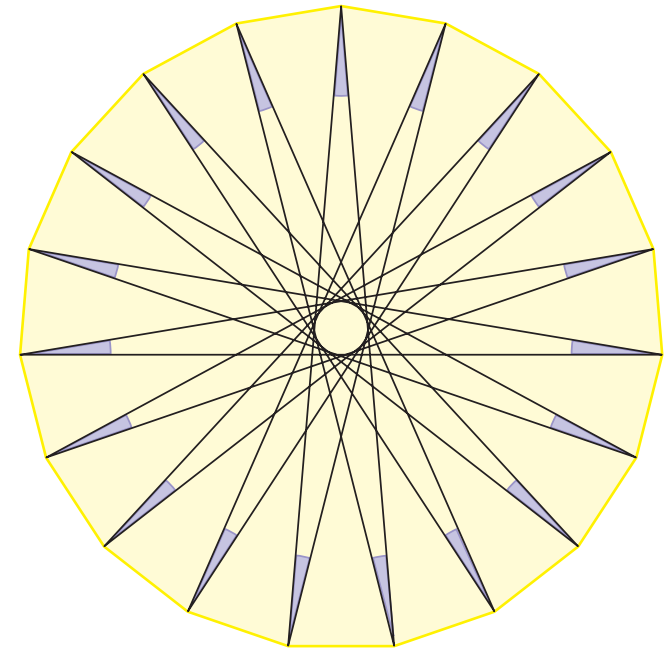

Pис. 9

что подходящую замкнутую 19-звенную ломаную можно нарисовать, обходя вершины правильного 19-угольника по этим диагоналям (рис.9).

Итак, при $n=19$ пример для замкнутой ломаной нарисовать получилось, а для многоугольника - не вышло.

Вернемся к равенству (2). Угол $\left(180^{\circ}-\alpha_{i}\right)$ дополняет угол $\alpha_{i}$ до $180^{\circ}$, это смежный с ним угол, внешний угол многоугольника. Действительно, направление обхода изменяется как раз на величину этого внешнего угла. Это верно и для углов, бо́льших $180^{\circ}$ : если $\varphi>180^{\circ}$, то угол величиной $\left(180^{\circ}-\varphi\right)$ по-прежнему можно назвать смежным с ним. Правда, его величина будет отрицательной, но отрицательные величины углов нам уже несколько привычны. На рисунке 10 серым цветом отмечены смежные углы к углу $\varphi$ для двух случаев: $\varphi<180^{\circ}$ и $\varphi>180^{\circ}$. Так что $S-$ это сумма внешних углов многоугольника. А у мно-
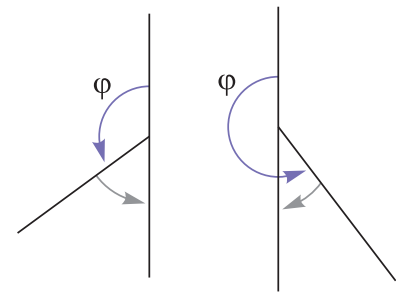

Рис. 10 гоугольников (как выпуклых, так и невыпуклых) она, как известно, равна $360^{\circ}$ ! В точности равна, а не только кратна $360^{\circ}$, как это выходит для обычных замкнутых ломаных.

Тем самым получается, что направление обхода совершит ровно один оборот против часовой стрелки. Для выпуклых многоугольников это вполне очевидно (см. рис.5). Как раз рассмотрением такой картинки и можно доказать теорему о сумме внешних углов выпуклого многоугольника (т.е. тот факт, что она равна $360^{\circ}$ ). Правда, для невыпуклых многоугольников это утверждение уже совсем не очевидно, и проще доказать формулу суммы углов (внешних или внутренних) невыпуклого многоугольника иначе. Здесь мы не будем приводить это доказательство. С ним читатель может познакомиться, например, в статьях Н.Васильева и В.Гутенмахера «Сумма углов» и «О разрезаниях многоугольника и теореме Эйлера» в «Кванте» №2 за 1988 год.

\section{Упражнения}

4. Найдите значение $S$ для замкнутой ломаной на рисунке 9 . Как зависит $S$ от направления обхода ломаной?

5. Приведите пример замкнутой самопересекающейся ломаной, для которой а) $S=360^{\circ}$; б) $S=0^{\circ}$.

\section{Улучшаем оценку}

Мы разобрались, что $S=360^{\circ}$, а тогда из равенства (2) получаем $180^{\circ} \cdot(a-b-2)=$ $=A-B$. Так как $0<A<10^{\circ} \cdot a$ и $0<B<$ $<10^{\circ} \cdot b$, то

$$
-10^{\circ} \cdot b<180^{\circ} \cdot(a-b-2)<10^{\circ} \cdot a .
$$

Поскольку $n=a+b$ нечетно, то и $a-b$ нечетно. Разберем 4 случая, каким оно может быть:

- $a-b \geq 5$, тогда $180^{\circ} \cdot 3<10^{\circ} \cdot a$, т.е. $n \geq a \geq 55$

- $a-b=3$, тогда $180^{\circ}<10^{\circ} \cdot a$, т.е. $a \geq 19$ и $b=a-3 \geq 16$, значит, $n \geq 19+16=35$;

- $a-b=1$, тогда $10^{\circ} \cdot b>180^{\circ}$, т.е. $b \geq 19$ и $a=b+1 \geq 20$, значит, $n \geq 19+20=39$; 
- $a-b \leq-1$, тогда $10^{\circ} \cdot b>180^{\circ} \cdot 3$, т.e. $n \geq b \geq 55$.

Итак, во всех случаях количество вершин не меньше 35: $M \geq 35$.

\section{Получаем пример}

Из оценки следует, что вариант $n=35$ возможен лишь при $a=19, b=16$. Это помогает придумать пример: 4 подряд маленьких угла (в порядке обхода границы многоугольника), затем большой угол, затем снова маленький и так далее маленькие углы чередуются с большими (рис.11).

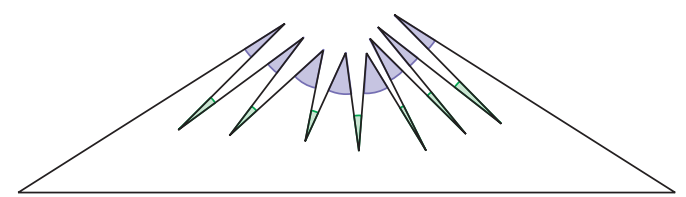

Рис. 11

Осталось показать, что устроенный таким образом пример действительно существует и нам не помешают какие-нибудь противоречия, на которые мы еще не обратили внимания.

Построение примера можно описать так. Возьмем треугольник, два угла которого меньше $10^{\circ}$, а третий - меньше $170^{\circ}$ (например: $\left.9^{\circ}, 9^{\circ}, 162^{\circ}\right)$. Больший его угол разрежем еще на 17 маленьких углов (на рисунке 12 их 8). Получится замкнутая ломаная, у которой часть звеньев друг на друга накладываются. В нем 19 маленьких углов и 16 углов в $360^{\circ}$ (на рисунке они отмечены красным). Осталось последовательно подвигать вершины, чтобы стороны перестали накладываться друг на друга, но при этом маленькие углы остались маленькими. Таким образом будет получен искомый многоугольник.

Итак, $M=35$. Задача решена.

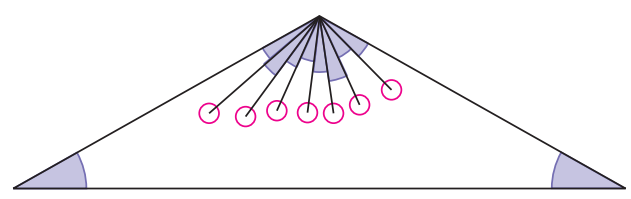

Напоследок приведем еще две интересные задачи на затронутые темы.

\section{Дополнительные задачи}

1 (В.Произволов, «Задачи на вырост»). Каждый из углов 19-угольника кратен $10^{\circ}$. Докажите, что у него найдутся две параллельные стороны.

2. Какое наибольшее количество острых углов может быть а) в выпуклом, б) в произвольном $n$-угольнике?

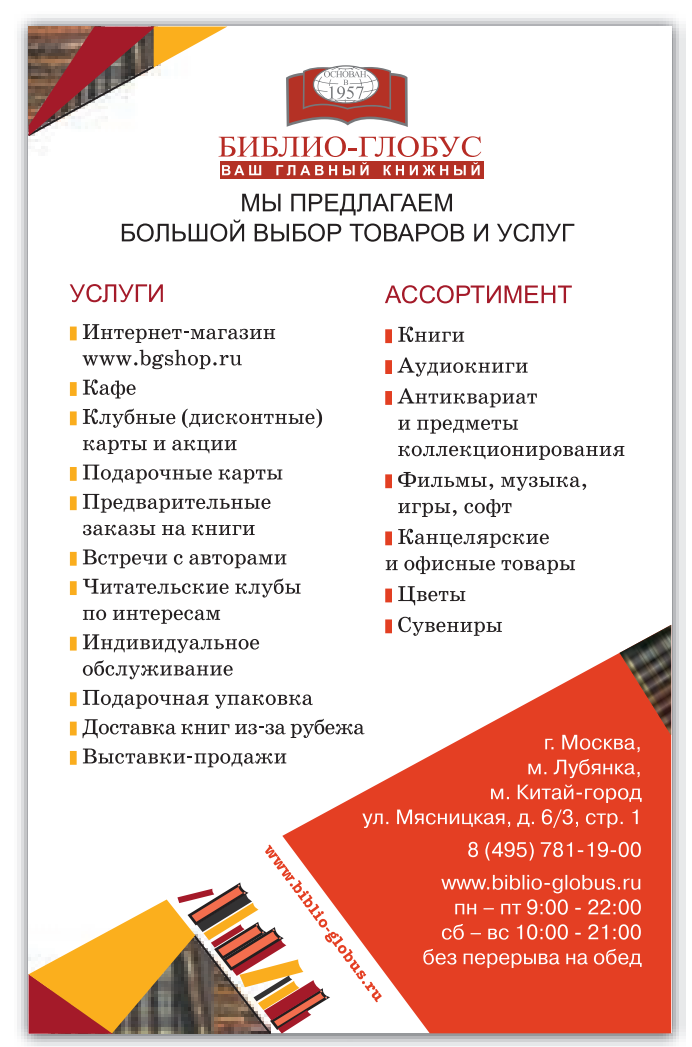

